\title{
STABILITY OF NEGATIVE EQUILIBRIUM OF A NON-LINEAR DIFFERENCE EQUATION
}

\section{ERKAN TAŞDEMIR and YÜKSEL SOYKAN}

Kirklareli University

Pinarhisar Vocational School of Higher Education

39300, Kirklareli

Turkey

e-mail: erkantasdemir@hotmail.com

Department of Mathematics

Faculty of Art and Science

Bülent Ecevit University

67100, Zonguldak

Turkey

e-mail: yuksel_soykan@hotmail.com

\begin{abstract}
In this study, we will investigate global stability of negative equilibrium of difference equation

$$
x_{n+1}=x_{n-1} x_{n-3}-1, \quad n=0,1,2, \ldots,
$$

where the initial conditions are in the interval $(-1,0)$. We show that every solution of Equation (1.1) converges to the negative equilibrium $\frac{1-\sqrt{5}}{2}$.
\end{abstract}

2010 Mathematics Subject Classification: 39A10, 39A30.

Keywords and phrases: difference equations, negative equilibrium, stability.

Received January 31, 2018; Revised February 14, 2018

(C) 2018 Scientific Advances Publishers 


\section{Introduction and Preliminaries}

During the last years, althought many authors have focused on convergence of positive equilibrium point of solutions of difference equations ([1-10]), some authors prefer to study negative equilibrium of difference equations (see [11-13]).

In [10], we investigated the stability analysis of Equation (1.1). According to this, positive equilibrium of Equation (1.1) is unstable and negative equilibrium of Equation (1.1) is locally asymptotically stable.

This study aims to work the convergence of negative equilibrium of Equation (1.1).

Definition 1. A second order difference equation is an equation of the form

$$
x_{n+1}=f\left(x_{n}, x_{n-1}\right) ; \quad n=0,1,2, \ldots,
$$

for every set of the initial conditions $x_{0}, x_{-1} \in I$.

Theorem 2.1 ([7], p13). Let $[a, b]$ be an interval of real numbers and assume that

$$
f:[a, b] \times[a, b] \rightarrow[a, b]
$$

is a continious function satisfying the following properties:

(a) $f(x, y)$ is non-increasing in each of its arguments;

(b) If $(m, M) \in[a, b] \times[a, b]$ is a solution of the system

$$
f(m, m)=M \text { and } f(M, M)=m,
$$

then $m=M$.

Then Equation (2.1) has a unique equilibrium $\bar{x} \in[a, b]$ and every solution of Equation (2.1) converges to $\bar{x}$.

Equation (1.1) is equivalent to the following difference equation:

$$
y_{n+1}=1-y_{n-1} y_{n-3}, \quad n=1,2, \ldots,
$$


with change to variable $y_{n}=-x_{n}$. Thus, the initial conditions of (2.2) are $y_{-3}=-x_{-3}, y_{-2}=-x_{-2}, y_{-1}=-x_{-1}$, and $y_{0}=-x_{0}$.

Now, we take two subsequences $t_{n}=y_{2 n-1}$ and $w_{n}=y_{2 n}$ for $n \geq-1$. Therefore, we obtain that

$$
t_{n+1}=1-t_{n} t_{n-1} ; \quad t_{-1}, t_{0} \in I,
$$

and

$$
w_{n+1}=1-w_{n} w_{n-1} ; \quad w_{-1}, w_{0} \in I,
$$

with the positive equilibrium point $\bar{y}=\bar{t}=\bar{w}=\frac{\sqrt{5}-1}{2}$.

Besides, the interval $(0,1)$ is invariant for Equation $(2.2)$ as in [3] as Theorem 4.1.

\section{Main Results}

In this section, we study the convergence behaviour of negative equilibrium of Equation (1.1).

Theorem 3.1. Every solution of Equation (1.1) with the initial conditions in $(0,1)$ converges to the positive equilibrium $\bar{y}=(\sqrt{5}-1) / 2$.

Lemma 3.2. The equilibrium point $\bar{t}$ is a global attractor of Equation (2.3).

Proof. Let $g:[a, b]^{2} \rightarrow[a, b]$ is a function defined by

$$
g(u, v)=1-u \cdot v .
$$

Then

$$
\begin{gathered}
\frac{\partial g(u, v)}{\partial u}=-v<0 \\
\frac{\partial g(u, v)}{\partial v}=-u<0 .
\end{gathered}
$$

Therefore $g$ is non-increasing function in each its arguments. 
Now, we consider that $(m, M) \in[a, b]^{2}$ is a solution of the system $M=g(m, m)$ and $m=g(M, M)$. Hence, from Equation (2.3)

$$
\begin{aligned}
& M=1-m \cdot m, \\
& m=1-M \cdot M .
\end{aligned}
$$

Thus, we have

$$
M=m
$$

Then, the proof is completed.

Now, we investigate global behaviour of Equation (2.4).

Lemma 3.3. The equilibrium point $\bar{w}$ is a global attractor of Equation (2.4).

Proof. Let $g:[a, b]^{2} \rightarrow[a, b]$ is a function defined by

$$
g(u, v)=1-u \cdot v
$$

Then

$$
\begin{aligned}
& \frac{\partial g(u, v)}{\partial u}=-v<0, \\
& \frac{\partial g(u, v)}{\partial v}=-u<0 .
\end{aligned}
$$

Therefore $g$ is non-increasing function in each its arguments.

Now, we consider that $(m, M) \in[a, b]^{2}$ is a solution of the system $M=g(m, m)$ and $m=g(M, M)$. Hence, from Equation (2.4)

$$
\begin{aligned}
& M=1-m \cdot m, \\
& m=1-M \cdot M .
\end{aligned}
$$

Thus, we have

$$
M=m \text {. }
$$

Then, the proof is completed. 
Proof of Theorem 3.1. We consider Lemma 3.2 and Lemma 3.3. We also know that sequences $t_{n}$ and $w_{n}$ are subsequences of $y_{n}$. So, $y_{n}$ converges to the positive equilibrium $\bar{y}$. Due to the fact that $\bar{y}$ is positive equilibrium of Equation (2.2), Equation (1.1) converges to negative equilibrium point. So, the proof is completed.

\section{Numerical Examples}

In this section, we demonstrate some numerical examples about main result.

Example 1. If the initial conditions are $x_{-3}=-0.1, x_{-2}=-0.3$, $x_{-1}=-0.5$, and $x_{0}=-0.9$, then negative equilibrium $\bar{x}$ is global attractor of Equation (1.1). Figure 1 shows this status.

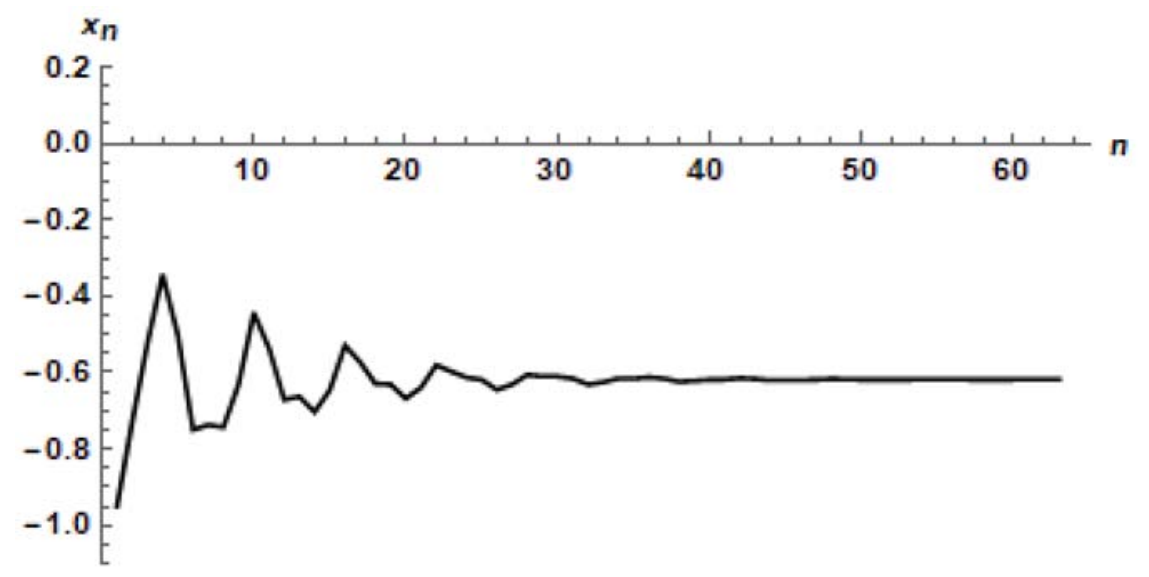

Figure 1. Plot of Equation (1.1) with initial conditions $x_{-3}=-0.1$, $x_{-2}=-0.3, x_{-1}=-0.5$, and $x_{0}=-0.9$. 
Example 2. If the initial conditions are $x_{-3}=-0.3, x_{-2}=-0.8$, $x_{-1}=-0.6$, and $x_{0}=-0.2$, then negative equilibrium $\bar{x}$ is global attractor of Equation (1.1). Figure 2 illustrates this status.

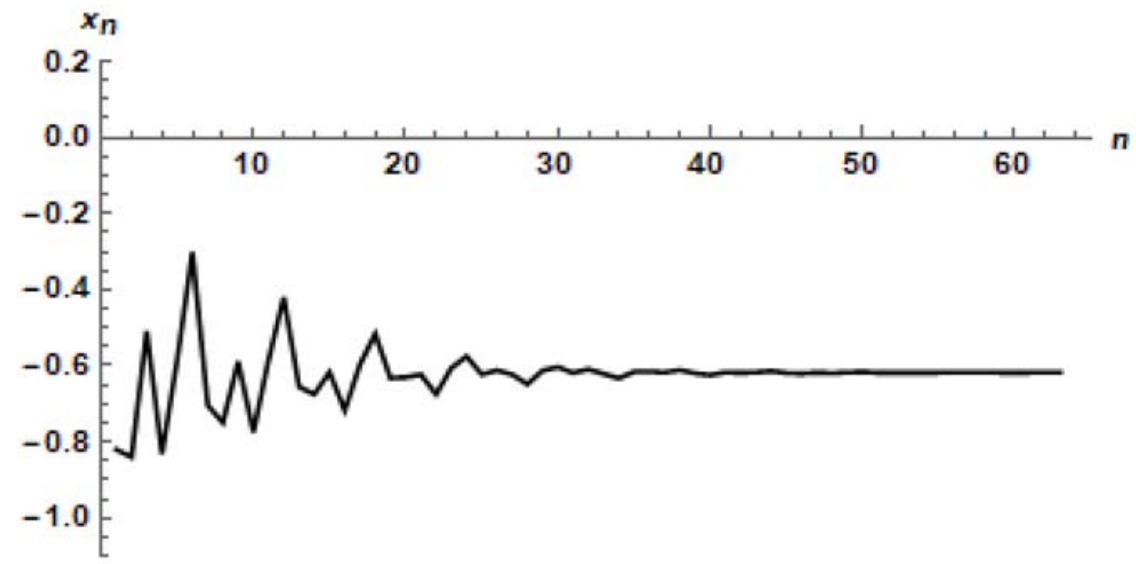

Figure 2. Plot of Equation (1.1) with initial conditions $x_{-3}=-0.3$, $x_{-2}=-0.8, x_{-1}=-0.6$, and $x_{0}=-0.2$.

\section{References}

[1] S. Elaydi, An Introduction to Difference Equations, Springer Science \& Business Media, New York, 2005.

[2] M. Göcen and M. Güneysu, The global attractivity of some rational difference equations, Journal of Computational Analysis and Applications 25(7) (2018), 1233-1243.

[3] C. M. Kent and W. Kosmala, On the nature of solutions of the difference equation $x_{n+1}=x_{n} x_{n-3}-1$, International Journal of Nonlinear Analysis and Applications 2(2) (2011), 24-43.

DOI: https://doi.org/10.22075/IJNAA.2011.91

[4] C. M. Kent, W. Kosmala and S. Stevic, Long-term behavior of solutions of the difference equation $x_{n+1}=x_{n-1} x_{n-2}-1$, Abstract and Applied Analysis (2010), Article ID 152378, 17 pages.

DOI: http://dx.doi.org/10.1155/2010/152378 
[5] C. M. Kent, W. Kosmala and S. Stevic, On the difference equation $x_{n+1}=x_{n} x_{n-2}-1$, Abstract and Applied Analysis (2011), Article ID 815285, 15 pages.

DOI: http://dx.doi.org/10.1155/2011/815285

[6] C. M. Kent, W. Kosmala, M. A. Radin and S. Stevic, solutions of the difference equation $x_{n+1}=x_{n} x_{n-1}-1$, Abstract and Applied Analysis (2010), Article ID 469683, 13 pages.

DOI: http://dx.doi.org/10.1155/2010/469683

[7] M. R. S. Kulenovic and G. Ladas, Dynamics of Second Order Rational Difference Equations: With Open Problems and Conjectures, Chapman \& Hall/CRC, Boca Raton, London, 2001.

[8] S. Stevic and B. Iricanin, Unbounded solutions of the difference equation $x_{n}=x_{n-l} x_{n-k}-1$, Abstract and Applied Analysis (2011), Article ID 561682, 8 pages.

DOI: http://dx.doi.org/10.1155/2011/561682

[9] E. Taşdemir and Y. Soykan, On the periodicies of the difference equation $x_{n+1}=x_{n} x_{n-1}+\alpha$, Karaelmas Science and Engineering Journal 6(2) (2016), 329-333.

DOI: http://dx.doi.org/10.7212\%2Fzkufbd.v6i2.333

[10] E. Taşdemir and Y. Soykan, Long-term behavior of solutions of the non-linear difference equation $x_{n+1}=x_{n-1} x_{n-3}-1$, General Mathematics Notes 38(1) (2017), 13-31.

[11] X. M. Jia and G. M. Tang, Global attractivity of a higher-order nonlinear difference equation, International Journal of Difference Equations 5(1) (2010), 95-101.

[12] C. Gan, X. Yang and W. Liu, Global behavior of $x_{n+1}=\left(\alpha+\beta x_{n-k}\right) /\left(\gamma+x_{n}\right)$, Discrete Dynamics in Nature and Society (2013), Article ID 963757, 5 pages.

DOI: http://dx.doi.org/10.1155/2013/963757

[13] Y. Wang, Y. Luo and Z. Lu, Convergence of solutions of $x_{n+1}=x_{n} x_{n-1}-1$, Applied Mathematics E-Notes 12 (2012), 153-157. 